\title{
$\mathbf{J}|\mathbf{A}| \mathbf{C} \mid \mathbf{S}$ \\ COMMUNICATIONS
}

Published on Web 06/04/2002

\section{A Smart Adhesive Joint: Entropic Control of Adhesion at a Polymer/Metal Interface}

\author{
Sureurg Khongtong and Gregory S. Ferguson* \\ Departments of Chemistry and Materials Science \& Engineering, Lehigh University, \\ Bethlehem, Pennsylvania 18015-3172
}

Received October 26, 2001

Although adhesives, adhesion-promoters, and adhesion-enhancing treatments have been developed to meet a tremendous number of varied applications, ${ }^{1,2}$ designing systems in which adhesion itself is responsive to changes in environmental conditions would offer opportunities for active control of adhesion over time (e.g., in applications such as antifouling, barrier films, or cell adhesion). ${ }^{3}$ We report here a polymer/metal interface that displays reversible, temperature-dependent adhesion arising from rubber elasticity in the interfacial region of the polymer. Our strategy for coupling rubber elasticity and interfacial adhesion involved constructing a system that required extension of polymer chains out of randomcoil conformations to bring adhesion-promoting functional groups to the polymer/metal interface. As a result, the entropic loss associated with chain extension would provide a restoring force for removing these groups from the interface. The opposition of this entropic force to enthalpically favorable chemical interactions at the polymer/metal interface would, in turn, provide the temperature dependence of adhesion.

Oxidation of the surface of cross-linked 1,4-polybutadiene (1,4PBD) with aqueous permanganate introduces carboxylic acid and other functional groups ${ }^{4,5}$ within the interfacial region. Although polymer surfaces usually reconstruct against water to maximize hydrogen bonding at the interface, ${ }^{6,7}$ we have demonstrated previously that this surface-modified elastomer reconstructs reversibly against water as a function of temperature, to produce a hydrophilic surface (hydrogen-bonding groups in contact with the water) at low temperature but a hydrophobic one at higher temperature. ${ }^{4}$ We expected this material to behave analogously against aluminum because of the well-established affinity of carboxylic-acid groups for the native oxide of this metal. ${ }^{7}$

Cross-linked polymer films were prepared by mixing 1,4-PBD (36\% cis, $55 \%$ trans, and $9 \% 1,2-; M_{\mathrm{w}}=420000 \mathrm{~g} / \mathrm{mol}$ ) with 0.02 phr of dicumyl peroxide and curing at $150{ }^{\circ} \mathrm{C}$ under 125 psi for 84 min ( 8 half-lives). ${ }^{8}$ The cured film (approximately $1 \mathrm{~mm}$ thick; $M_{\mathrm{c}}$ $\approx 30000 \mathrm{~g} / \mathrm{mol}$ ) was cut into strips having lateral dimensions of $\sim 10 \mathrm{~cm} \times 1 \mathrm{~cm}$. The unbound fraction - polymer chains not incorporated into the network - was extracted by swelling the strips in toluene. ${ }^{4}$ This step ensured that reconstruction at the polymer surface would be due only to the movement of chains that were part of the cross-linked network. One of the broad surfaces on each sample was oxidized with a basic aqueous solution of $\mathrm{KMnO}_{4}$ for 50 min at room temperature, as described previously, ${ }^{4}$ to introduce carboxylic acid and other functional groups within the interfacial region. As a result of this oxidation, the contact angle of water (pH 1) dropped from $84-88^{\circ}$ to $66-72^{\circ} .{ }^{10}$ Aluminum strips (99.5\%; thickness $\sim 0.007 \mathrm{~mm}$ ) were cut to dimensions of $\sim 13 \mathrm{~cm}$ $\times 1 \mathrm{~cm}$ and sonicated in $75 \mathrm{~mL}$ of $3 \%(\mathrm{v} / \mathrm{v})$ aqueous detergent (Detergent 8; VWR) for 0.5 h. ${ }^{11}$ They were then thoroughly rinsed with deionized water and blown dry with a stream of nitrogen.

* To whom correspondence should be addressed. E-mail: gf03@lehigh.edu.

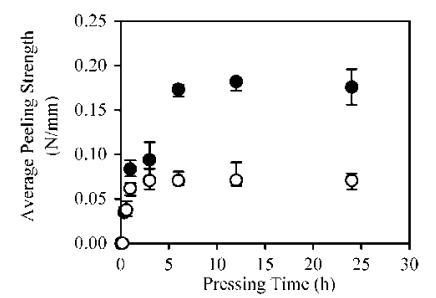

Figure 1. The growth of adhesion at unoxidized-1,4-PBD/aluminum $(O)$ and oxidized-1,4-PBD/aluminum (-) interfaces as a function of the pressing time at room temperature. The points represent average values measured on separate samples, and the error bars indicate maximum and minimum peeling strengths measured for that sample at that time.

Strips of the surface-modified polymer and aluminum were placed into contact, except at one end where the two surfaces were separated by $\sim 1 \mathrm{~cm}$ of Teflon tape to provide places to be gripped by a tensile adhesion tester. This specimen was placed between two rigid plates with another strip of Teflon tape between the polymer and its adjacent plate to prevent them from adhering one another. It was then pressed under $\sim 50$ psi in a hydraulic press at a specified temperature. After a specified amount of time, the pressure was released, and the sample was allowed to cool to room temperature. The amount of adhesion at the polymer/metal interface was then measured using a T-peel test performed on an Instron 5567 tensile machine with a 500-N loadcell. The test was run at room temperature with a peel rate of $20 \mathrm{~mm} / \mathrm{min}$ for all specimens, and the average peeling strengths reported were evaluated from the peeling load in the plateau region of the force-displacement plots using Instron Series IX software.

As shown in Figure 1, the limiting adhesion at the interface between the oxidized surface and aluminum was about 3 times higher than that for unoxidized 1,4-PBD. This large increase in adhesion at this interface is consistence with the anticipated chemical bonding between the oxidized-1,4-PBD and $\mathrm{Al} / \mathrm{Al}_{2} \mathrm{O}_{3}$ surfaces. ${ }^{8}$ The initial rate in the growth of adhesion was similar for both surfaces, though the adhesion for the oxidized sample continued to increase until it reached the steady-state level after 6 $\mathrm{h}$. The growth in adhesion of the unoxidized sample presumably reflects ingress of the polymer into the microscopic roughness of the aluminum surface, ${ }^{12}$ and the continued growth in adhesion of the oxidized sample is consistent with reconstruction of the polymer surface to bring carboxylic acid groups to the surface of the aluminum (oxide). To test the stability of the interfacial adhesion over time, several laminates were prepared and pressed under $\sim 50$ psi at room temperature for $18 \mathrm{~h}$. The pressure was then released, and samples were monitored for adhesion strength as a function of time at room temperature. The adhesion was monitored for separate samples, each aged for different lengths of time: $0,1,2,3$, and 7 days. We found that the adhesion of this interface was stable throughout this period, even in the absence of applied pressure. ${ }^{13}$ 


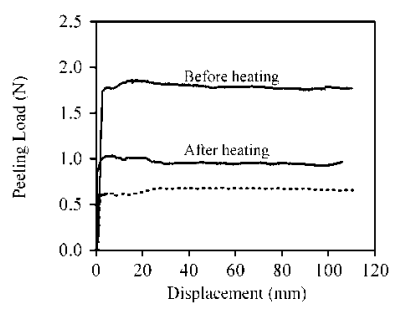

Figure 2. Adhesion at the oxidized-1,4-PBD/aluminum interface before and after heating at $80{ }^{\circ} \mathrm{C}$ for $15 \mathrm{~min}$. The heated specimen was quickly cooled to room temperature under a stream of nitrogen before measuring the adhesion. The dashed line shows the adhesion at the unoxidized-1,4$\mathrm{PBD} /$ aluminum interface, for comparison.

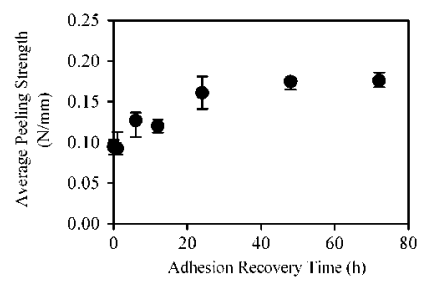

Figure 3. The kinetics of recovery of adhesion at an oxidized-1,4-PBD/ aluminum interface after samples heated at $80{ }^{\circ} \mathrm{C}$ for $15 \mathrm{~min}$ were subsequently equilibrated at room temperature.

To test our central hypothesis regarding "smart" temperatureresponsive adhesion in this system, we examined the strength of adhesion in laminated samples that had been equilibrated at room temperature and at elevated temperature. Two sets of oxidized1,4-PBD/Al samples were prepared in the same way and pressed at the same time under $\sim 50 \mathrm{psi}$ at room temperature for $18 \mathrm{~h}$. The adhesion strength for the first set of these samples was then measured, and the other set was pressed under the same pressure for an additional $15 \mathrm{~min}$, though at $80{ }^{\circ} \mathrm{C}$. After cooling to room temperature, the adhesion strength of these samples was also measured. Figure 2 shows representative force-displacement curves for T-peel tests for the different types of samples. The data for unoxidized-1,4-PBD against aluminum pressed at $\sim 50 \mathrm{psi}$ at room temperature, a system whose adhesion was found to be independent of temperature, ${ }^{14}$ are added for comparison. When equilibrated at $80{ }^{\circ} \mathrm{C}$, the strength of adhesion at the oxidized-1,4-PBD/Al interface dropped by $44 \%$ from its room-temperature value $(\sim 1.8 \mathrm{~N})$ to approximately $\sim 1.0 \mathrm{~N}$. This decrease in adhesion strength is analogous to the decrease in hydrophilicity displayed by this polymer surface when equilibrated against water at $80{ }^{\circ} \mathrm{C},{ }^{4}$ and is consistent with our hypothesis regarding the role of rubber elasticity at the interface. The adhesion level of the heated sample was only slightly higher than that of the unoxidized sample, indicating that few of the oxidized functional groups remained at the interface. The approach of the adhesion strength toward that of the unoxidized sample, while remaining above that value, suggested that the polymer and $\mathrm{Al}$ surfaces remained in contact and did not delaminate.

This change in the adhesion at the oxidized-1,4-PBD/Al interface was reversible; when a sample that had been heated at $80{ }^{\circ} \mathrm{C}(\sim 50$ psi, $15 \mathrm{~min}$ ) was allowed to equilibrate at room temperature under the same pressure, the interfacial adhesion slowly recovered and reached its initial steady-state level within about $40 \mathrm{~h}$ (Figure 3). In fact, this reversibility extended through several cycles of heating at $80{ }^{\circ} \mathrm{C}$ and cooling to room temperature. The slow increase in adhesion shown in Figure 3, relative to the faster increase observed when the interface was initially pressed (Figure 1), as well as the temperature independence of adhesion at the unmodified-1,4-PBD/ $\mathrm{Al}$ interface, also argues strongly against simple delamination being the mechanism responsible for the temperature-dependent changes in this system. It also argues against the possibility that the decrease in adhesion upon heating was due to "microdelamination" arising from entropic stress associated with deformation of polymer chains in the interfacial region as they ingress into the microstructure of aluminum surface.

In summary, the interface of a surface-modified 1,4-PBD and aluminum produced a strong adhesive joint at room temperature due to enthalpically favorable chemical interactions between the added functional groups and the surface of the metal substrate. On the basis of the behavior of this polymer against water, we hypothesized that migration of these functional groups into contact with the aluminum (oxide) would require polymer chains to extend out of their random coil conformations, thus reducing the entropy in that region of the polymer. At high temperature, in turn, these functional groups would be pulled away from the interface by the elastic restoring force induced by the entropic loss in the extended polymer chains. Consistent with these hypotheses, adhesion at the oxidized-1,4-PBD interface varied dramatically and reversibly with changes in temperature. This strategy of integrating the bulk and interfacial properties of a polymer, we believe, offers unique opportunities for the design of "smart" (responsive) systems.

Acknowledgment. We acknowledge support for these studies from the Office of Naval Research (ONR) and thank the Royal Thai Government for providing a graduate scholarship for S.K. We also thank Walailak University for providing S.K. the opportunity for a final review of this paper.

\section{References}

(1) Mittal, K. L. Ed. Polymer Surface Modification: Relevance to Adhesion; VSP BV: Zeist, The Netherlands, 1996. Mittal, K. L. Ed. Silanes and Other Coupling Agents; VSP BV: Zeist, The Netherlands, 1992. Schneberger, G. L., Ed., Adhesives in Manufacturing; Marcel Dekker: New York, 1983.

(2) Kumar, A.; Biebuyck, H.; Whitesides, G. M. Langmuir 1994, 10, 14981511. Maege, I.; Jaehne, E.; Henke, A.; Adler, H. P.; Bram, C.; Jung, C. Stratmann, M. Prog. Org. Coat. 1998, 34, 1-12. Zarnoch, K. P. J. Adhes Sci. Technol. 1994, 8, 501-509. Rohwerder, M.; Stratmann, M. MRS Bull. 1999, 24, 43-47. Aronoff, Y. G.; Chen, B.; Lu, G.; Seto, C.; Schwartz J.; Bernasek, S. L. J. Am. Chem. Soc. 1997, 119, 159-162. André, V.; Arefi, F.; Amouroux, J.; Lorang, G. Surf. Interface Anal. 1990, 16, $241-$ 245. Costin, R.; Nagel, W. Kautsch. Gummi Kunstst. 1999, 52, $188-$ 192. Reinartz, C.; Fuerbeth, W.; Stratmann, M. J. Anal. Chem. 1995, 353, 657-600. Mumbauer, P. D.; Carey, D. H.; Ferguson, G. S. Chem. Mater. 1995, 7, 1303-1314.

(3) Callow, M. E.; Callow, J. A.; Ista, L. K.; Coleman, S. E.; Nolasco, A. C.; Lopez, G. P. Appl. Environ. Microbiol. 2000, 66, 3249-3254. Zhang, S.; Wright, G.; Yang, Y. Biosens. Bioelectron. 2000, 15, 273-282. de Crevoisier, G.; Fabre, P.; Corpart, J.; Leibler, L. Science 1999, 285, 12461249. Ista, L. K.; Perez-Luna, V. H.; Lopez, G. P. Appl. Environ. Mocrobiol. 1999, 65, 1603-1609. Ista, L. K.; Lopez, G. P. J. Ind. Microbiol. Biotechnol. 1998, 20, 121-125.

(4) Khongtong, S.; Ferguson, G. S. J. Am. Chem. Soc. 2001, 123, 35883594.

(5) Carey, D. H.; Ferguson, G. S. Macromolecules 1994, 27, 7254-7266.

(6) Bergbreiter, D. E. In Field Responsive Polymers: Electroresponsive Photoresponsive, and Responsive Polymers in Chemistry and Biology, Khan, I. M., Harrison, J. S., Eds.; American Chemical Society: Washington, DC, 1999; pp 301-310. Ferguson, G. S.; Whitesides, G. M. In Modern Approaches in Wettability: Theory and Applications; Schrader, M. E., Leob, G. I., Eds.; Plenum: New York, 1992; pp 143-175.

(7) Chen, W.; McCarthy, T. J. Macromolecules 1999, 32, 2342-2347. Xie, X.; Gengenbach, T. R.; Griesser, H. J. J. Adhes. Sci. Technol. 1992, 6, $1411-1431$.

(8) Rancourt, J. D.; Hollenhead, J. B.; Taylor, L. T. J. Adhes. 1993, 40, $267-$ 285. Ulren, L.; Hjertberg, T.; Ishida, H. J. Adhes. 1990, 31, 117-136.

(9) Brandrup, J.; Immergut, E. H.; Grulke, E. Polymer Handbook, 4th ed.; John Wiley \& Sons: New York, 1999.

(10) A more complete characterization of this material was reported in ref 4

(11) Westray, W. K. In Adhesives in Manufacturing; Schneberger, G. L., Ed.; Marcel Dekker: New York, 1983; pp 427-436.

(12) The values of surface roughness $\left(R_{\mathrm{rms}}\right)$, measured by atomic force microscopy (AFM), were $\sim 250 \AA$ for the aluminum surfaces and $\sim 60 \AA$ for unoxidized- and oxidized-1,4-PBD surfaces. Lee, H. J. Adhes. 1994, $46,15-38$.

(13) The average peeling strength for all samples was about $0.17 \mathrm{~N} / \mathrm{mm}$.

(14) The average peeling strength at the unmodified-1,4-PBD/aluminum interface before and after heating was about the same $(\sim 0.06 \mathrm{~N} / \mathrm{mm})$. This control experiment rules out residual stress as a possible mechanism for the changes in adhesion for the oxidized samples.

JA017396E 